\title{
Fusion Pores Live on the Edge
}

\author{
Edgar M. Blokhuis, Massimo D’Agostino, Andreas Mayer, and H. Jelger Risselada*
}

Cite This: J. Phys. Chem. Lett. 2020, 11, 1204-1208

Read Online

ABSTRACT: Biological transmission of vesicular content occurs by opening of a fusion pore. Recent experimental observations have illustrated that fusion pores between vesicles that are docked by an extended flat contact zone are located at the edge (vertex) of this zone. We modeled this experimentally observed scenario by coarse-grained molecular simulations and elastic theory. This revealed that fusion pores experience a direct attraction toward the vertex. The size adopted by the resulting vertex pore strongly depends on the apparent contact angle between the adhered vesicles even in the absence of membrane surface tension. Larger contact angles substantially increase the equilibrium size of the vertex pore. Because the cellular membrane fusion machinery actively docks membranes, it facilitates a collective expansion of the contact zone and increases the contact angle. In this way, the fusion machinery can drive expansion of the fusion pore by free energy equivalents of multiple tens of $k_{\mathrm{B}} T$ from a distance and not only through the fusion proteins that reside within the fusion pore.

B iological membrane fusion proceeds via the opening of a fusion pore to release vesicular cargoes that are vital for many biological processes, including exocytosis, intracellular trafficking, fertilization, and viral entry. Cryo-electron tomography (cryo-ET) observations of in vivo fusion events in synapses ${ }^{1}$ and $3 \mathrm{D}$-live cell microscopy of yeast cells ${ }^{2}$ suggest that fusion is preceded by close apposition of the two membranes, which for larger vesicles $(>100 \mathrm{~nm})$ results in the formation of an extended flat contact or docking zone. ${ }^{3}$ Subsequent fusion is thought to occur at the highly curved membrane perimeter of the contact zone-the vertex., Indeed, cryo-ET of reconstituted mitochondrial fusion as well as fluorescence microscopy studies of yeast vacuole fusion revealed fusion pores that are located at the vertex; see refs 2 and 5 and Figure 1.

Pores Are Intrinsically Attracted toward the Vertex. The physical principle underlying "vertex pores" can be illustrated from a coarse-grained molecular simulation of two curved membrane sheets that are being connected by a centrally located fusion pore (see the SI for details). Rather than symmetrizing membrane curvature at both sides of the pore, such a system "escapes" into a highly asymmetric shape by adopting an off-centered location-a vertex pore (see Figure 1c). Attraction toward the edge is in our example explained by a strong, favorable reduction in membrane curvature at the left side of the pore, whereas the curvature at the opposing side remains rather conserved. Symmetry breaking, i.e., location near the edge, thus provides a net free energy gain. A fusion pore-if not being nucleated at the vertex-will thus become captured at the vertex after having diffused to this location. However, quite in contrast to the stereotypical model of an axial symmetric fusion pore, a "vertex pore" is not characterized by axial symmetry because of the varying membrane curvature along its circumference. The consequences of such an altered architecture/symmetry on pore size have remained unexplored.

A large amount of both theoretical work (e.g., continuum elastic models and molecular simulations $)^{6-15}$ and experimental observations (e.g., patch-clamp experiments and cryoEM tomography) $)^{2,5,16-19}$ have substantially advanced our understanding of the structure, composition, location, dynamics, and energetics of fusion pores. Irrespective of their topology, fusion pores in living cells are likely to be neither protein channels nor purely lipid but are probably proteolipidic hybrid structures. ${ }^{16-19}$ Fusion proteins such as SNAREs and associated tether complexes are integrated into them and play an active role in the opening and dynamics of the fusion pore via steric, entropic, and electrostatic forces. ${ }^{19-22}$ An expansive radial force on the pore originates from the crowding of proteins at the pore's circumference. The proteins must be part of the pore in order to influence pore size in this way. The structure of a vertex pore additionally depends on the (effective) contact angle of the contact zone (Figure 2). Here, we will illustrate that this contact angle determines the equilibrium size of the vertex pore. The important consequence of this principle is that docking mediators such as mitofusins, SNAREs, and associated tether complexes, which determine the size of the contact zone and hence the contact

Received: December 3, 2019

Accepted: January 16, 2020

Published: January 16, 2020 

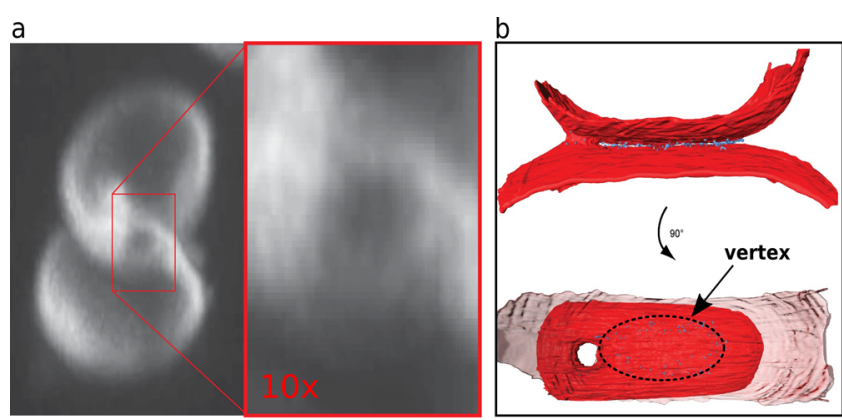

C
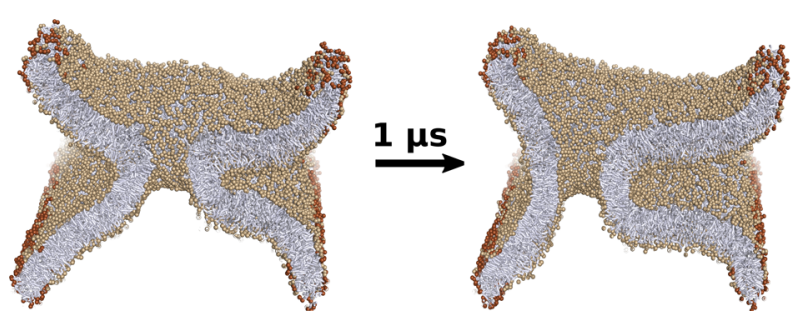

Figure 1. Vertex fusion pores. (a) Stacked tomography imaging of the in vivo fusion reaction between two yeast vacuoles (adapted from ref 2). (b) Cryo-electron tomography of the adhesion zone formed in reconstituted mitochondrial fusion (adapted from ref 5). (c) Centrally located pore formed between two curved lipid membrane sheets undergoing spontaneous symmetry breaking in a coarse-grained molecular dynamics simulation (see the Supporting Information Movie). The dark brown colored beads indicate immobilized beads, which spatially constrain the free membrane ends (see the SI and Figure S4). angle, can not only influence fusion pore dynamics when being directly integrated into the pore, but they can also impose an additional distal influence on the pore.

Vertex Attractions Inherently Facilitate Pore Expansion. Because it is virtually impossible to experimentally discern the intrinsic contribution of the contact angle from a potentially present membrane tension, we reconstructed a coarse-grained molecular simulation model of an edge fusion pore located at the perimeter of an extended docking zone (see Figure 2a). Tensionless membrane conditions were ensured by breaking the periodicity along the $x$-dimension, which enabled the membranes to freely adopt area. To study a specific effective contact angle $\theta$, we employed an external field to enforce the membranes to adopt a desired angle with respect to the contact zone (see the SI for technical details). In this procedure, we started from a system comprised of two flat membranes where a small stable fusion pore (area of $\sim 5 \mathrm{~nm}^{2}$ ) was already present. Then, we gradually increased the contact angle. From this trajectory, we extracted different contact angles, which we independently studied by a long equilibrium run $(2 \mu \mathrm{s})$ at a constant contact angle. Figure $2 \mathrm{~b}, \mathrm{c}$ shows the equilibrium size of the fusion pore as a function of contact angle. Surprisingly, from $\theta>30^{\circ}$, the area of the metastable pore steeply increases and reaches up to 9 -fold in size $(5 \rightarrow 45$ $\left.\mathrm{nm}^{2}\right)$ at $\theta=45^{\circ}$. Conveniently, the free energy $F$ required to expand a symmetric fusion pore $\left(\theta=0^{\circ}\right)$ to a radius $R$ can be extracted from our molecular simulations by enforcing a radial expansion of the pore via an applied external field and extracting the average, responsive force, $\frac{\mathrm{d} F}{\mathrm{~d} R}$, acting against that
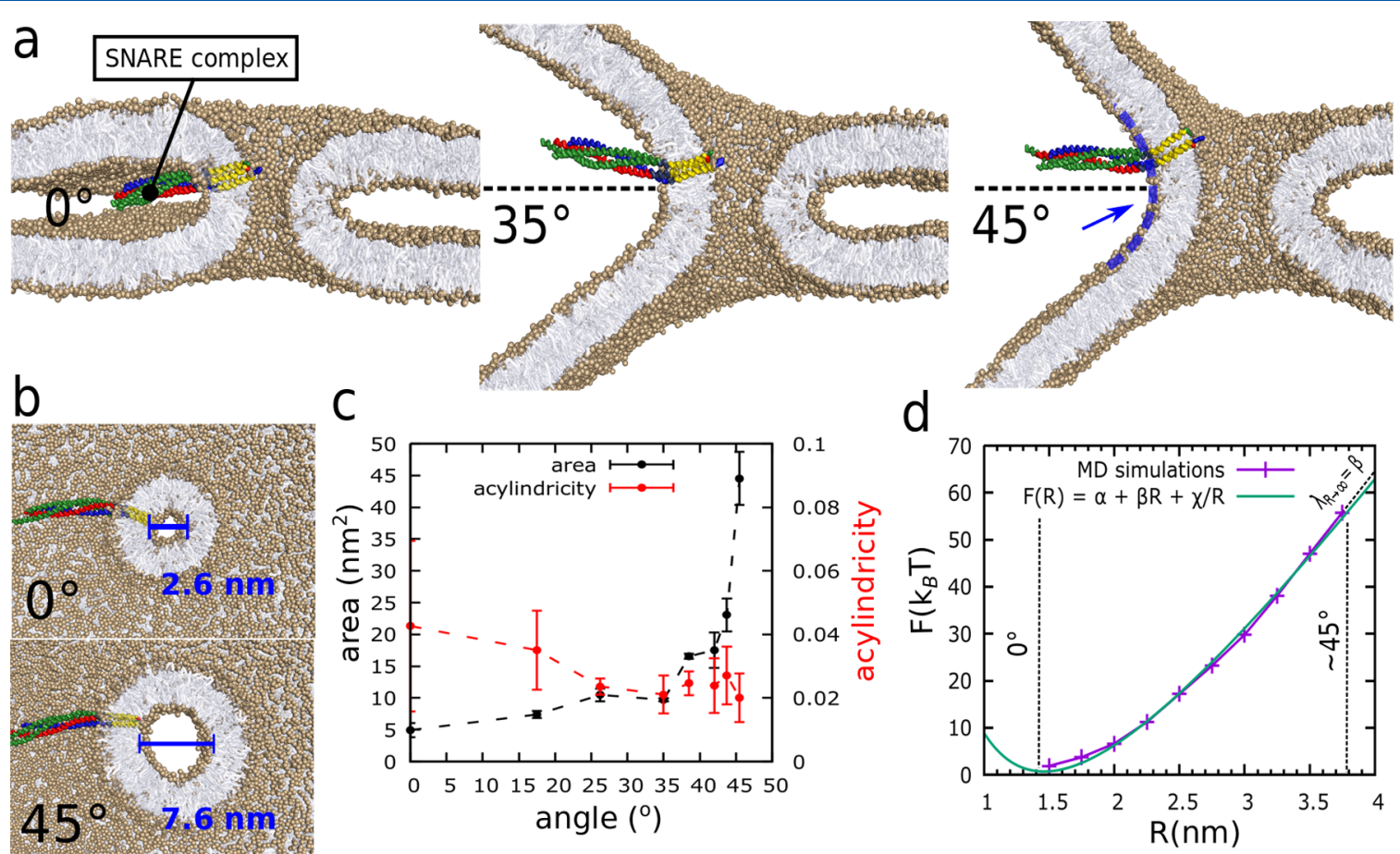

Figure 2. Molecular dynamics simulations of the vertex fusion pore under different contact angles. The depicted SNARE complex indicates the length scale. (a) Final states of the system (after $2 \mu \mathrm{s}$ ) under effective contact angles of 0,35 , and $45^{\circ}$. The blue dashed line indicates the induced axial asymmetry of the pore's circumference. (b) Corresponding top view of the fusion pore. (c) Area and acylindricity $(\in[0,1])$ of the fusion pore as a function of the contact angle. Acylindricity values close to zero indicate that the pore adopts a perfect circular shape. The error bars are the standard errors derived by ensemble block averaging. (d) Free energy cost associated with the radial expansion of a free, symmetric pore $\left(\theta=0^{\circ}\right.$ ). Adopting a pore area of $45 \mathrm{~nm}^{2}$ (a radius of $\simeq 3.8 \mathrm{~nm}$ ), i.e., the native pore size at $\theta=45^{\circ}$, requires a free energy equivalent of about $55 k_{\mathrm{B}} T$. The shape of $F(R)$ is qualitatively described by the function $F(R)=\alpha+\beta R+\frac{\gamma}{R}$. 

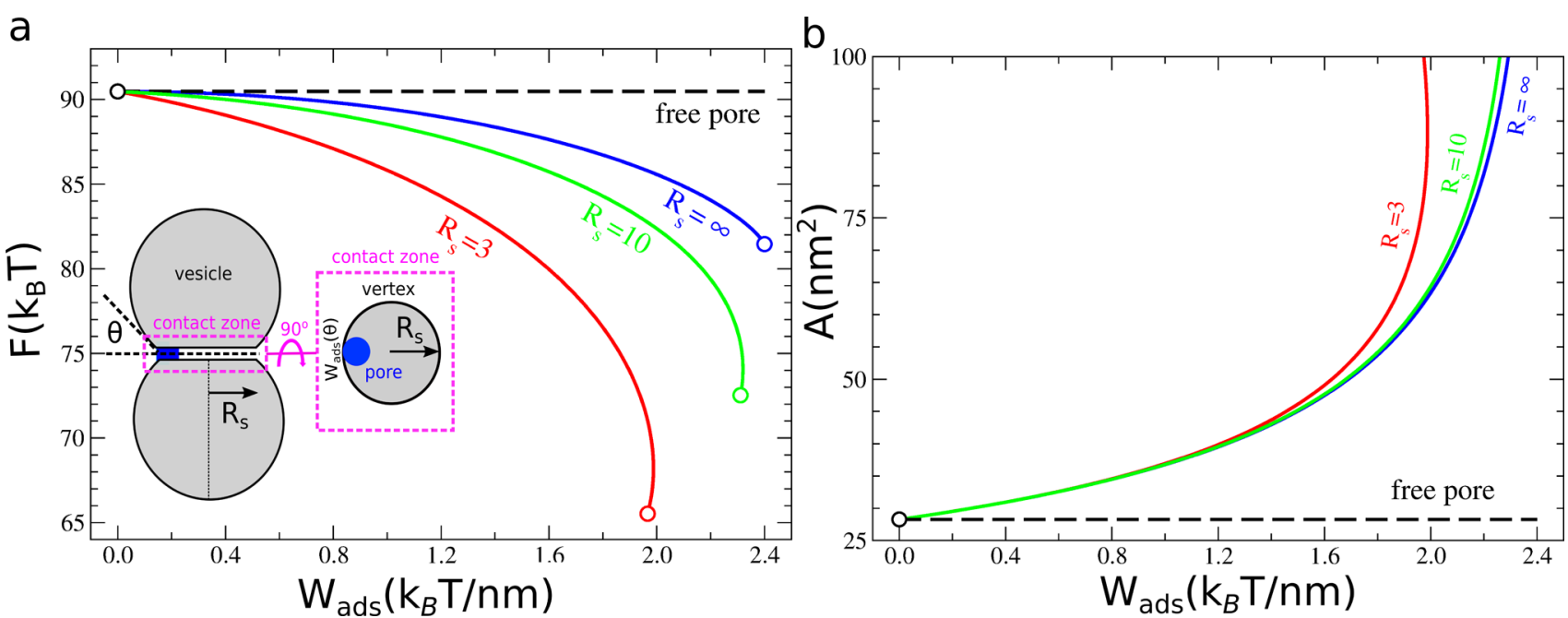

Figure 3. Vertex attractions drive pore expansion. (a) Free energy of the vertex pore as a function of edge attractions. (b) Corresponding equilibrium area of the pore. The dashed black line ("free pore") illustrates the value of a common fusion pore (no vertex attractions). The size of the contact zone is in reduced units: $R_{\mathrm{s}}=3(12.7 \mathrm{~nm})$ and $10(42.4 \mathrm{~nm})$.

field (see Figure 2d). Intriguingly, a 9-fold expansion of a symmetric fusion pore $\left(\theta=0^{\circ}\right)$ would require a free energy equivalent of more than $50 k_{\mathrm{B}} T$. Thus, the vertex provides a substantial driving force for pore expansion. Alternatively, pore size may be enhanced by binding of a voluminous protein complex such as, e.g., the HOPS complex near the fusion pore. ${ }^{23}$ Within such a scenario, expansion occurs when the effective spherical size of a nearby complex is above a size of $\sim 20 \mathrm{~nm}$ (Figure S9), which can be attained by common SNARE-associated protein complexes. ${ }^{24,25}$

Thermodynamic Description of a Fusion Pore. A fusion pore adopts a thermodynamically stable size because of a force balance along its circumference, $2 \pi R$. The free energy of the axially symmetric fusion pore can be expanded in terms of $R$ as $F(R)=\alpha+\beta R+\frac{\gamma}{R}+\frac{\delta}{R^{2}} \ldots(R>0)$. Constant terms within the free energy $(\alpha)$ can be omitted. This expansion directly follows from the fact that the contribution of one of its principle radii to the free energy must vanish when the pore becomes large. In that regime, $F(R)$ linearly increases with the length of the interface, $F(R) \propto R(R \gg 0)$, because the line tension, being thermodynamically defined as $\lambda_{R}=\frac{1}{2 \pi} \frac{\mathrm{d} F}{\mathrm{~d} R}$, becomes constant; therefore, $\lambda_{R \rightarrow \infty}=\frac{\beta}{2 \pi}$. The competition between the contractive linear term $\left(\lambda_{R \rightarrow \infty}\right)$ and the expansive rigidity term $(\mathrm{s})$ gives rise to a force balance: a free energy minimum that determines the equilibrium size of the pore. In regular membrane pores where the contractive forces are dominant, such a free energy minimum is extremely shallow and pores are either unstable or short-lived. ${ }^{26}$ Figure $2 \mathrm{~d}$ illustrates that inclusion of the first "rigidity" term $\left(\frac{\gamma}{R}\right)$ suffices to qualitatively describe the free energy associated with fusion pore expansion. This justifies the thermodynamic description of a three-dimensional fusion pore by an enclosed contour (a two-dimensional vesicle), whose size and shape are understood from a balance between the contractive force, $\lambda_{R \rightarrow \infty, \theta=0}$, and the bending rigidity, $\kappa_{2 \mathrm{D}}=\frac{\gamma}{2 \pi}$ (see the SI).

Why Vertex Attractions Impose Expansion. Because a larger contact angle enables the vertex pore to relax its curvature stress at least on one side, it translates into a stronger vertex attraction. This attraction is effectively modeled by the force, $W_{\text {ads }}$. Vertex pore formation is analogous to the adhesion of a two-dimensional vesicle (the pore) to a curved substrate (the vertex). ${ }^{14,27,28}$ The shape equations corresponding to this variational wetting problem were solved numerically (see the SI). Figure 3 illustrates that edge attractions push the force balance toward larger pore sizes, in correspondence with the molecular simulations. The vertex attraction $W_{\text {ads }}$ locally compensates for the intrinsic contractive force acting on the pore's circumference, being $\lambda_{R \rightarrow \infty, \theta=0}$. Evidently, even a local, asymmetric release of bending energy already suffices to expand the pore. By consequence, axially symmetric fusion pores are expected to expand when the distance between the two opposed membranes increases because of a global, symmetric release in bending energy. ${ }^{7}$ Decreasing the radius of the vertex $R_{\mathrm{s}}$ below a microscopic size-thereby better matching the pore's native circular shape-further increases pore growth. This suggests that the expected pore growth is stronger in smaller vesicles, such as SUVs and synaptic vesicles, because of a smaller contact zone, given that the (apparent) contact angle between the adhered vesicles is similar. Finally, modeling the vertex as an attractive hard wall induces deformation of the pore when interacting with the vertex (see the SI). The observation of a circular pore in the molecular simulations therefore rather indicates that vertex interactions are soft.

Vertex Pores in Vivo. A remaining question is whether vertex attractions also significantly affect pore expansion in vivo, for which we should expect contact angles $\theta>30^{\circ}$. On the basis of the microscopic observation of docked yeast vacuoles, ${ }^{2}$ we estimated a contact angle of about $50^{\circ}$ (see Figure S10). However, it is challenging to directly relate the microscopically observed contact angle in experiments with the here-reported nanoscopic, apparent contact angle. ${ }^{29}$ Fortunately, these nanoscopic contact angles are directly transferable into a concomitant adhesion free energy per unit area (a surface tension), $\sigma$, via the relationship $\sigma_{\theta}=\frac{\kappa}{2 R_{\theta}{ }^{2}},{ }^{29}$ with $\kappa$ being the bending modulus $\left(\sim 20 k_{\mathrm{B}} T\right)$ and $R_{\theta}$ the radius of adhesionthe membrane curvature (radius) at the point of intersection with the contact zone (see Figures S11 and S12). We find $R_{\theta=35}$ $\approx 80 \mathrm{~nm} \rightarrow \sigma_{\theta=35}=1.6 \times 10^{-3} k_{\mathrm{B}} T / \mathrm{nm}^{2}$ and $R_{\theta=45} \approx 40 \mathrm{~nm} \rightarrow$ 
$\sigma_{\theta=45}=5.8 \times 10^{-3} k_{\mathrm{B}} T / \mathrm{nm}^{2}$. Thus, we predict that the proteinmediated adhesion/docking of membranes must yield $1.6 \times$ $10^{-3} k_{\mathrm{B}} T / \mathrm{nm}^{2}$ or $6.6 \times 10^{-3} \mathrm{mN} / \mathrm{m}\left(1 k_{\mathrm{B}} T / \mathrm{nm}^{2}=4.114 \mathrm{mN} /\right.$ $\mathrm{m}$ at $293 \mathrm{~K}$ ) to substantially contribute to the free energy of the fusion pore via vertex interactions. This value represents a common adhesive interaction between lipid membranes ${ }^{30}$ and can be experimentally determined via micropipette aspiration. $^{30,31}$ Because the direct contribution of this adhesive interaction to the pore's free energy is approximately $\sigma A,{ }^{7,12}$ its intrinsic effect on equilibrium pore size is small $\left(<1 k_{\mathrm{B}} T\right)$.

A compelling amount of evidence suggests that fusion proteins actively contribute to the expansion of a formed fusion pore. ${ }^{19-22}$ Such a pore expansion can be driven by entropic repulsions between fusion proteins integrated within the pore. ${ }^{20}$ As shown here, vertex attractions offer an additional and perhaps surprising mechanism, by which also distal fusion proteins can substantially contribute to the expansion of the fusion pore via a collective expansion of the contact zone. Furthermore, our work strongly suggests that the "black holes" recently observed in yeast vacuole fusion assays, i.e., subnanometer sized fusion pores that are too small to allow passage of soluble dye molecules, are not explained by their observed vertex location. ${ }^{2}$ Because vertex attractions are rather expected to increase the size of a fusion pore, black holes must be due to the presence of an additional, dominant contractive force on the fusion pore in docked yeast vacuoles, for example, the presence of electrostatic attractions among net charged lipid species, protein residues, and ions inside of the pore. ${ }^{16}$ Finally, popular experimental assays for studying the conductance of the fusion pore are based on the fusion reaction between nanodisks and membranes. ${ }^{32}$ Nanodisks are comprised of a peptide- or polymer-capped free membrane edge that introduces a spatially heterogeneous membrane environment analogous to the vertex of the docking zone. Therefore, the free energy of the fusion pore may depend on its location within the disk. Because "edge attractions" increase pore size regardless of the edge's structural nature (Figure 3), it is a relevant question whether the fusion pore formed in larger nanodisks $(>20 \mathrm{~nm}$ ) preferably locates near the rim because of edge attractions or whether it adopts a central location because of edge repulsions.

\section{ASSOCIATED CONTENT}

\section{SI Supporting Information}

The Supporting Information is available free of charge at https://pubs.acs.org/doi/10.1021/acs.jpclett.9b03563.

Methods and additional simulations and calculations (PDF)

Movie showing how a centrally located fusion pore breaks its symmetry (MP4)

\section{AUTHOR INFORMATION}

\section{Corresponding Author}

H. Jelger Risselada - Leiden Institute of Chemistry (LIC), Leiden University, 2333 CD Leiden, The Netherlands; Department of Theoretical Physics, Georg-August University of Goettingen, 37077 Goettingen, Germany; 이이이.org/00000003-1410-6570; Phone: +49 55139 5995; Email: hrissel@ gwdg.de; Fax: +49551399631

\section{Authors}

Edgar M. Blokhuis - Leiden Institute of Chemistry (LIC), Leiden University, 2333 CD Leiden, The Netherlands

Massimo D'Agostino - Department of Molecular Medicine and Medical Biotechnology, University of Naples Federico II, 80138 Naples, Italy

Andreas Mayer - Département de Biochimie, Université de Lausanne, CH-1015 Epalinges, Switzerland

Complete contact information is available at: https://pubs.acs.org/10.1021/acs.jpclett.9b03563

\section{Notes}

The authors declare no competing financial interest.

\section{ACKNOWLEDGMENTS}

H.J.R. acknowledges the Life@nano excellence initiative (State of Lower Saxony, Germany), the NWO Vidi scheme (the Netherlands), and the DFG grant RI 2791/2-1 (Germany) for funding. A.M. has been supported by the SNSF (31003A_179306). The HLRN Berlin/Hannover \& NWO SURFsara (The Netherlands) are acknowledged for computational resources.

\section{REFERENCES}

(1) Imig, C.; Min, S.-W.; Krinner, S.; Arancillo, M.; Rosenmund, C.; Südhof, T. C.; Rhee, J.; Brose, N.; Cooper, B. H. The Morphological and Molecular Nature of Synaptic Vesicle Priming at Presynaptic Active Zones. Neuron 2014, 84, 416-431.

(2) D'Agostino, M.; Risselada, H. J.; Endter, L. J.; Comte-Miserez, V.; Mayer, A. SNARE-mediated membrane fusion arrests at pore expansion to regulate the volume of an organelle. EMBO J. 2018, 37, e99193.

(3) Hernandez, J. M.; Stein, A.; Behrmann, E.; Riedel, D.; Cypionka, A.; Farsi, Z.; Walla, P. J.; Raunser, S.; Jahn, R. Membrane Fusion Intermediates via Directional and Full Assembly of the SNARE Complex. Science 2012, 336, 1581-1584.

(4) Wang, L.; Seeley, E.; Wickner, W.; Merz, A. J. Vacuole Fusion at a Ring of Vertex Docking Sites Leaves Membrane Fragments within the Organelle. Cell 2002, 108, 357-369.

(5) Brandt, T.; Cavellini, L.; Kühlbrandt, W.; Cohen, M. M. A mitofusin-dependent docking ring complex triggers mitochondrial fusion in vitro. eLife 2016, DOI: 10.7554/eLife.14618.

(6) Nanavati, C.; Markin, V.; Oberhauser, A.; Fernandez, J. The exocytotic fusion pore modeled as a lipidic pore. Biophys. J. 1992, 63, $1118-1132$

(7) Chizmadzhev, Y.; Cohen, F.; Shcherbakov, A.; Zimmerberg, J. Membrane mechanics can account for fusion pore dilation in stages. Biophys. J. 1995, 69, 2489-2500.

(8) Jackson, M. Minimum Membrane Bending Energies of Fusion Pores. J. Membr. Biol. 2009, 231, 101-115.

(9) Grafmüller, A.; Shillcock, J.; Lipowsky, R. The Fusion of Membranes and Vesicles: Pathway and Energy Barriers from Dissipative Particle Dynamics. Biophys. J. 2009, 96, 2658-2675.

(10) Yoo, J.; Jackson, M. B.; Cui, Q. A Comparison of CoarseGrained and Continuum Models for Membrane Bending in Lipid Bilayer Fusion Pores. Biophys. J. 2013, 104, 841-852.

(11) Ryham, R. J.; Ward, M. A.; Cohen, F. S. Teardrop shapes minimize bending energy of fusion pores connecting planar bilayers. Phys. Rev. E 2013, DOI: 10.1103/PhysRevE.88.062701.

(12) Long, R.; Hui, C.-Y.; Jagota, A.; Bykhovskaia, M. Adhesion energy can regulate vesicle fusion and stabilize partially fused states. $J$. R. Soc., Interface 2012, 9, 1555-1567.

(13) Gao, L.; Lipowsky, R.; Shillcock, J. Tension-induced vesicle fusion: pathways and pore dynamics. Soft Matter 2008, 4, 1208-1214. 
(14) Risselada, H. J.; Smirnova, Y.; Grubmüller, H. Free Energy Landscape of Rim-Pore Expansion in Membrane Fusion. Biophys. J. 2014, 107, 2287-2295.

(15) Sharma, S.; Lindau, M. Molecular mechanism of fusion pore formation driven by the neuronal SNARE complex. Proc. Natl. Acad. Sci. U. S. A. 2018, 115, 12751-12756.

(16) Han, X.; Wang, C. T.; Bai, J.; Chapman, E. R.; Jackson, M. B. Transmembrane Segments of Syntaxin Line the Fusion Pore of $\mathrm{Ca}^{+}$ -Triggered Exocytosis. Science 2004, 304, 289-292.

(17) Fang, Q.; Berberian, L. W.; Gong, K.; Hafez, I.; Sørensen, J. B.; Lindau, $M$. The role of the $C$ terminus of the SNARE protein SNAP25 in fusion pore opening and a model for fusion pore mechanics. Proc. Natl. Acad. Sci. U. S. A. 2008, 105, 15388-15392.

(18) Bao, H.; Goldschen-Ohm, M.; Jeggle, P.; Chanda, B.; Edwardson, J. M.; Chapman, E. R. Exocytotic fusion pores are composed of both lipids and proteins. Nat. Struct. Mol. Biol. 2016, 23, 67-73.

(19) Bao, H.; Das, D.; Courtney, N. A.; Jiang, Y.; Briguglio, J. S.; Lou, X.; Roston, D.; Cui, Q.; Chanda, B.; Chapman, E. R. Dynamics and number of trans-SNARE complexes determine nascent fusion pore properties. Nature 2018, 554, 260-263.

(20) Wu, Z.; Thiyagarajan, S.; O'Shaughnessy, B.; Karatekin, E. Regulation of Exocytotic Fusion Pores by SNARE Protein Transmembrane Domains. Front. Mol. Neurosci. 2017, DOI: 10.3389/ fnmol.2017.00315.

(21) Wu, Z.; Bello, O. D.; Thiyagarajan, S.; Auclair, S. M.; Vennekate, W.; Krishnakumar, S. S.; O'Shaughnessy, B.; Karatekin, E. Dilation of fusion pores by crowding of SNARE proteins. eLife 2017, DOI: $10.7554 /$ eLife.22964.

(22) Mostafavi, H.; Thiyagarajan, S.; Stratton, B. S.; Karatekin, E.; Warner, J. M.; Rothman, J. E.; O'Shaughnessy, B. Entropic forces drive self-organization and membrane fusion by SNARE proteins. Proc. Natl. Acad. Sci. U. S. A. 2017, 114, 5455-5460.

(23) D’Agostino, M.; Risselada, H. J.; Lürick, A.; Ungermann, C.; Mayer, A. A tethering complex drives the terminal stage of SNAREdependent membrane fusion. Nature 2017, 551, 634.

(24) Brocker, C.; Kuhlee, A.; Gatsogiannis, C.; kleine Balderhaar, H. J.; Honscher, C.; Engelbrecht-Vandre, S.; Ungermann, C.; Raunser, S. Molecular architecture of the multisubunit homotypic fusion and vacuole protein sorting (HOPS) tethering complex. Proc. Natl. Acad. Sci. U. S. A. 2012, 109, 1991-1996.

(25) Chou, H.-T.; Dukovski, D.; Chambers, M. G.; Reinisch, K. M.; Walz, T. CATCHR, HOPS and CORVET tethering complexes share a similar architecture. Nat. Struct. Mol. Biol. 2016, 23, 761-763.

(26) Ting, C. L.; Awasthi, N.; Muller, M.; Hub, J. S. Metastable Prepores in Tension-Free Lipid Bilayers. Phys. Rev. Lett. 2018, 120, 120.

(27) Seifert, U. Adhesion of vesicles in two dimensions. Phys. Rev. A: At., Mol., Opt. Phys. 1991, 43, 6803-6814.

(28) Shi, W.; Feng, X. Q.; Gao, H. Two-dimensional model of vesicle adhesion on curved substrates. Acta Mech. Sin. 2006, 22, 529535.

(29) Seifert, U.; Lipowsky, R. Adhesion of vesicles. Phys. Rev. A: At., Mol., Opt. Phys. 1990, 42, 4768-4771.

(30) Sun, Y.; Lee, C.-C.; Huang, H. W. Adhesion and Merging of Lipid Bilayers: A Method for Measuring the Free Energy of Adhesion and Hemifusion. Biophys. J. 2011, 100, 987-995.

(31) Warner, J. M.; Karatekin, E.; O'Shaughnessy, B. Model of SNARE-Mediated Membrane Adhesion Kinetics. PLoS One 2009, 4, e6375.

(32) Karatekin, E. Toward a unified picture of the exocytotic fusion pore. FEBS Lett. 2018, 592, 3563-3585. 